\title{
Anti-inflammatory action of ethanolic extract of Ramulus mori on the BLT2-linked cascade
}

\author{
Geun-Soo Park ${ }^{1}$, Jeong-Keun Kim ${ }^{2}$ E Jae-Hong Kim ${ }^{1, *}$ \\ ${ }^{1}$ Department of Biotechnology, College of Life Sciences and Biotechnology, Korea University, Seoul 02841, ${ }^{2}$ Department of Chemical \\ Engineering and Biotechnology, Korea Polytechnic University, Shihung 15073, Korea
}

\begin{abstract}
Mulberry tree twigs (Ramulus mori) contain large amounts of oxyresveratrols and have traditionally been used as herbal medicines because of their anti-inflammatory properties. However, the signaling mechanism by which $R$. mori exerts its anti-inflammatory action remains to be elucidated. In this study, we observed that $R$. mori ethanol extracts (RME) exerted an inhibitory effect on the lipopolysaccharide (LPS)-induced production of the pro-inflammatory cytokine interleukin-6 (IL-6) in Raw264.7 macrophage cells. Additionally, RME inhibited IL-6 production by blocking the leukotriene $B_{4}$ receptor-2 (BLT2)-dependent-NADPH oxidase 1 (NOX1)-reactive oxygen species (ROS) cascade, leading to anti-inflammatory activity. Finally, RME suppressed the production of the BLT2 ligands LTB4 and 12(S)-HETE by inhibiting the p38 kinase-cytosolic phospholipase $A_{2}-5$-/12-lipoxygenase cascade in LPS-stimulated Raw264.7 cells. Overall, our results suggest that RME inhibits the 'BLT2 ligand-BLT2'-linked autocrine inflammatory axis, and that this BLT2-linked cascade is one of the targets of the anti-inflammatory action of $R$. mori. [BMB Reports 2016; 49(4): 232-237]
\end{abstract}

\section{INTRODUCTION}

The mulberry tree belongs to the genus Morus, and it has been widely cultivated in eastern Asia and used in herbal medicines (1). Mulberry extracts contain large amounts of stilbenes, including oxyresveratrol (2,3',4,5'-tetrahydroxy-trans-stilbene; OXY) (2). OXY exhibits anti-oxidant, anti-hyperlipidemic and anti-inflammatory activities (3-5). Most studies of mulberry extracts have primarily focused on the leaves and root cortices of this tree, and little is known about the activity of twigs (called Ramulus mori) (6-8). Recently, several reports have suggested

*Corresponding author. Tel: +82-2-3290-3452; Fax: +82-2-32903938; E-mail: jhongkim@korea.ac.kr

http://dx.doi.org/10.5483/BMBRep.2016.49.4.002

Received 6 January 2016, Revised 13 January 2016, Accepted 16 February 2016

Keywords: BLT2, IL-6, Ramulus mori, Raw264.7 cells, ROS that $R$. mori extracts contain high levels of OXY and have anti-inflammatory properties (9-11). However, there is currently little information about the signaling mechanism underlying the anti-inflammatory activity induced by $R$. mori.

Leukotriene $\mathrm{B}_{4}$ receptor-2 (BLT2) is a G protein-coupled receptor for pro-inflammatory lipid mediators such as leukotriene $\mathrm{B}_{4}\left(\mathrm{LTB}_{4}\right)$ and 12(S)-hydroxyeicosatetraenoic acid (12(S)HETE) (12). Recent reports suggest that BLT2 is implicated in several inflammatory human diseases including asthma (13, 14), rheumatoid arthritis (15), cancer (16-18), and inflammatory bowel disease (19). Moreover, in inflammatory diseases, BLT2 has been closely associated with the generation of reactive oxygen species (ROS) via NADPH oxidases (NOXs) $(13,20)$. In a previous study, we demonstrated that the BLT2-NOX1-ROS cascade mediates LPS-induced IL-6 production (21). Despite the implications of BLT2 being an inflammatory mediator, a natural agent that inhibits BLT2 has not yet been discovered.

In this study, we prepared an ethanolic extract of $R$. mori ethanol (RME), and found that RME suppressed the LPS-induced IL-6 production by down-regulating the BLT2-linked cascade. Additionally, treatment with RME suppressed the synthesis of the BLT2 ligands $\mathrm{LTB}_{4}$ and 12(S)-HETE by blocking a p38-cytosolic phospholipase $\mathrm{A}_{2}$ (CPLA $)$-5-lipoxygenase (5-LO)/ 12-lipoxygenase (12-LO) signaling cascade. Together, our results suggested that the 'BLT2 ligand-BLT2'-linked autocrine axis is a target of the anti-inflammatory action of $R$. mori.

\section{RESULTS AND DISCUSSION}

\section{RME suppresses the LPS-induced IL-6 production in} Raw264.7 cells

IL-6 exerts various pro-inflammatory effects on many cell types, and high levels of IL- 6 have been associated with the pathogenesis of diverse inflammatory diseases $(22,23)$. Here, we investigated the anti-inflammatory effects of RME on LPS-stimulated IL-6 production in Raw264.7 macrophage cells. Our initial observations had shown that IL-6 production dramatically and time-dependently increased after LPS treatment $(100 \mu \mathrm{g} / \mathrm{ml})$ in Raw264.7 cells (Fig. 1A and B). Before studying the anti-inflammatory activities of RME, we determined its cytotoxicity in Raw264.7 cells. Raw264.7 cells were treated with

ISSN: 1976-670X (electronic edition)

Copyright (C) 2016 by the The Korean Society for Biochemistry and Molecular Biology

(c) This is an open-access article distributed under the terms of the Creative Commons Attribution Non-Commercial License (http://creativecommons.org/licenses/by-nc/4.0) which permits unrestricted non-commercial use, distribution, and reproduction in any medium, provided the original work is properly cited. 
A

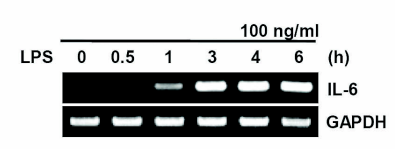

C

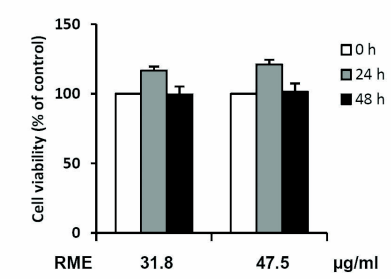

B

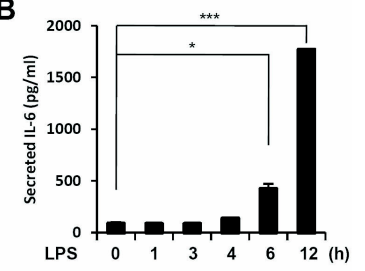

D

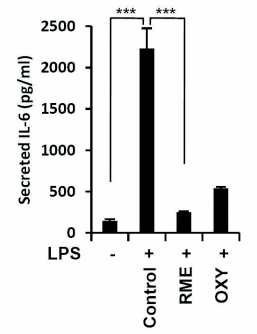

A

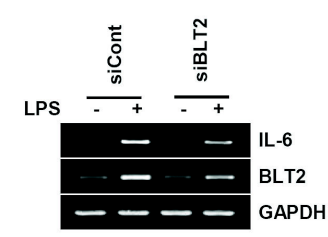

C

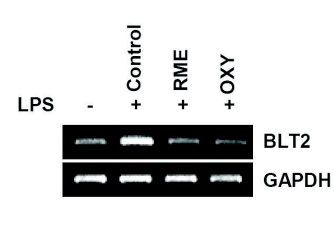

B

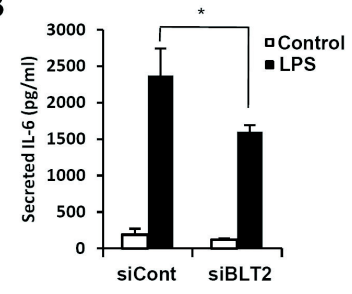

D

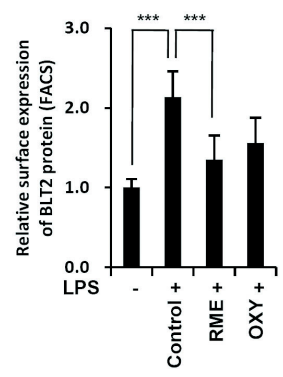

Fig. 1. RME suppresses LPS-induced IL-6 production in Raw264.7 cells. (A) Raw264.7 cells were stimulated with LPS (100 ng/ml) for the indicated times, and total RNA was subsequently subjected to RT-PCR analysis. (B) The amount of IL-6 released into the culture medium was measured by ELISA. (C) Raw264.7 cells were treated with RME (31.8 or $47.5 \mu \mathrm{g} / \mathrm{ml}$ ) for $0 \mathrm{~h}, 24 \mathrm{~h}$ or $48 \mathrm{~h}$. Cell viability was determined using an MTT assay. (D) Raw264.7 cells were incubated for $30 \mathrm{~min}$ with RME $(47.5 \mu \mathrm{g} / \mathrm{ml})$ or OXY $(7.3$ $\mu \mathrm{g} / \mathrm{ml}$ ) followed by $12 \mathrm{~h}$ of incubation in the presence or absence of LPS. Subsequently, the IL- 6 released into the culture medium was measured. All of the quantitative data are expressed as the mean \pm the SD, from three independent experiments. ${ }^{*} \mathrm{P}<0.05$, $* * * \mathrm{P}<0.005$.

different concentrations of RME (31.8 and $47.5 \mu \mathrm{g} / \mathrm{ml})$ for $48 \mathrm{~h}$ and evaluated by an MTT assay. As illustrated in Fig. 1C, RME did not exhibit any cytotoxicity. Next, we examined whether RME exhibited any inhibitory effects on LPS-induced IL-6 production in Raw264.7 cells. We observed that treatment with RME clearly suppressed the LPS-induced IL-6 production in Raw264.7 cells (Fig. 1D). OXY was used as a control, and the OXY treatment also suppressed LPS-induced IL-6 production, albeit less potently than RME (Fig. 1D). Thus, these results suggest that RME exerts its anti-inflammatory effects by suppressing the IL-6 production in Raw264.7 cells.

\section{RME inhibits LPS-induced BLT2 up-regulation in Raw264.7 cells}

Recent studies have implicated the function of BLT2 as a pro-inflammatory mediator in various inflammatory diseases, such as asthma (13, 14), arthritis (15), and arteriosclerosis (24). In previous studies, BLT2 up-regulation was shown to contribute to LPS-induced IL-6 production $(17,21)$. Indeed, siRNAmediated depletion of BLT2 resulted in significant inhibition of IL-6 expression in LPS-treated Raw264.7 cells (Fig. 2A and B). Therefore, we evaluated whether BLT2 is one of the targets of the anti-inflammatory activity of RME. We demonstrated that RME clearly suppressed LPS-induced BLT2 mRNA and protein
Fig. 2. RME inhibits LPS-induced BLT2 up-regulation in Raw264.7 cells. (A) Raw264.7 cells were transfected with control (siCont) or BLT2 (siBLT2) siRNAs. After $24 \mathrm{~h}$, the cells were incubated in the presence or absence of LPS for $4 \mathrm{~h}$, and the total RNA was isolated for RT-PCR analysis. (B) The Raw264.7 cells were transfected with control or BLT2 siRNAs, incubated for $24 \mathrm{~h}$ and then stimulated with LPS for $12 \mathrm{~h}$, after which the IL-6 released into the cell culture medium was measured. (C) Raw264.7 cells were incubated for $30 \mathrm{~min}$ with RME $(47.5 \mu \mathrm{g} / \mathrm{ml})$ or OXY $(7.3 \mu \mathrm{g} / \mathrm{ml})$ and then incubated for $4 \mathrm{~h}$ in the presence or absence of LPS $(100 \mathrm{ng} / \mathrm{ml})$. Subsequently, total RNA was isolated and subjected to RT-PCR analysis. (D) Raw264.7 cells were incubated for 30 min with RME or OXY treatment and subsequently incubated for $12 \mathrm{~h}$ in the presence or absence of LPS after the cells has been subjected to FACS analysis for BLT2 protein levels. All of the quantitative data are expressed as the mean \pm the SD, based on three independent experiments. $* P<0.05, * * * P<0.005$.

expression (Fig. 2C and D). OXY was used as a control, and OXY treatment also suppressed LPS-induced BLT2 expression (Fig. 2C and D). Together, these results suggest that RME attenuates the expression of BLT2, thus contributing to the suppression of IL-6 production.

\section{RME inhibits the LPS-induced NOX1-ROS cascade in Raw264.7 cells}

ROS generation is involved in a variety of pathological and inflammatory responses. For example, inflammatory stimulants (such as LPS) induce the generation of ROS, and LPS-induced ROS generation in macrophages is dependent on NADPH oxidase $(N O X)(25,26)$. We previously reported that NOX-derived ROS functions as downstream mediators of BLT2 in various cell types $(13,20,21,27)$, leading us to investigate whether RME attenuates the BLT2-dependent NOX-ROS cascade in LPS-activated Raw264.7 cells. RME clearly suppressed the LPS-induced ROS generation in a concentration-dependent 
manner, and exhibited anti-oxidative activity (Fig. 3A). Additionally, RME suppressed LPS-induced NOX1 up-regulation (Fig. 3B). OXY was used as a control, and OXY treatment also suppressed the LPS-induced ROS generation and NOX1 up-regulation (Fig. 3A and B). In contrast to NOX1, NOX2 and NOX4 were not up-regulated by LPS treatment (data not shown). Consistent with previous findings, NOX1 depletion by siRNA transfection resulted in significant inhibition of LPS-induced ROS generation and IL-6 expression (Figs. 3C-E). Additionally, we observed that LPS-induced NOX1 expression was greatly decreased by siRNA-mediated depletion of BLT2 in Raw264.7 cells (Fig. 3F). Together, these results suggest that RME down-regulates the BLT2-dependent NOX1-ROS cascade, thereby inhibiting IL-6 production in Raw264.7 cells.

\section{RME inhibits LPS-induced BLT2 ligand production in Raw264.7 cells}

BLT2 is activated by direct interaction with specific ligands, such as $\mathrm{LTB}_{4}$ and 12 (S)-HETE, on the cell surface (12). The synthesis of $\mathrm{LTB}_{4}$ and $12(\mathrm{~S})$-HETE from arachidonic acid (AA) is catalyzed by 5-lipoxygenase (5-LO) and 12-lipoxygenase (12-LO), respectively (28). Previously, we have reported that the levels of $\mathrm{LTB}_{4}$ and $12(\mathrm{~S})$-HETE are significantly increased by LPS in macrophages (21). To examine whether RME affects the production of the BLT2 ligands $\mathrm{LTB}_{4}$ and $12(\mathrm{~S})-\mathrm{HETE}$, we assessed the levels of these ligands. LPS-enhanced $\mathrm{LTB}_{4}$ and 12(S)-HETE expression were markedly inhibited by RME treatment (Fig. 4A). OXY was used as a control, and OXY treatment also inhibited LPS-induced production of the BLT2 ligands, albeit less potently than RME (Fig. 4A). Additionally, we observed that RME treatment inhibited the LPS-induced up-regulation of 5-LO and 12-LO in Raw264.7 cells (Fig. 4C). CPLA2 is the rate-limiting enzyme responsible for the release of $\mathrm{AA}$ from membrane phospholipids $(29,30)$, and $\mathrm{CPLA}_{2}$ activation was assessed by $\mathrm{Ser}^{505}$ phosphorylation, which was clearly increased by LPS treatment (Fig. 4C). Moreover, RME treatment significantly inhibited the LPS-induced stimulation of $\mathrm{CPLA}_{2}$ activity and $\mathrm{CPLA}_{2}$ phosphorylation in Raw264.7 cells (Fig. 4B and C). Previously, it has been suggested that p38 kinase activation is required for the LPS-induced activation of $\mathrm{CPLA}_{2}$ (31).
A

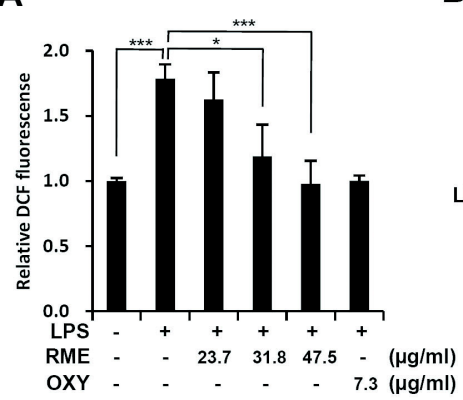

D

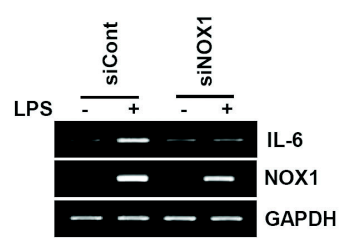

B

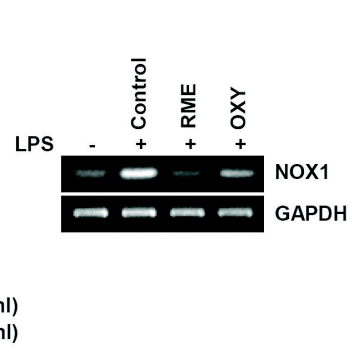

E

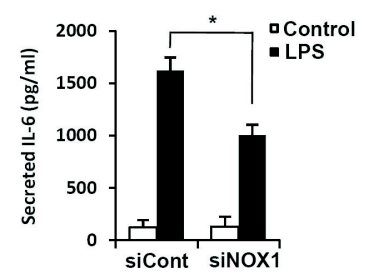

C

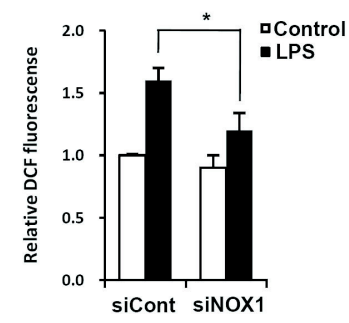

$\mathbf{F}$

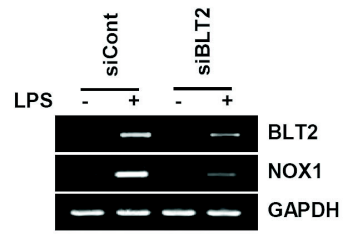

Fig. 3. RME inhibits the LPS-induced NOX1-ROS cascade in Raw264.7 cells. (A) Raw264.7 cells were incubated for 30 min with RME or OXY, followed by $1 \mathrm{~h}$ incubation in the presence or absence of LPS (100 ng/ml). 2',7'-dichlorofluorescein diacetate (DCF-DA; $10 \mu \mathrm{M})$ was added to the culture for 20 min during the final incubation, and intracellular ROS was subsequently measured using flow cytometric analysis of DCF fluorescence. (B) Raw264.7 cells were incubated for $30 \mathrm{~min}$ with RME $(47.5 \mu \mathrm{g} / \mathrm{ml})$ or OXY $(7.3 \mu \mathrm{g} / \mathrm{ml})$, followed by $4 \mathrm{~h}$ incubation in the presence or absence of LPS. Subsequently, total RNA was isolated and subjected to RT-PCR analysis. (C) The cells were transfected with NOX1 (siNOX) or control siRNA (siCont), incubated for $24 \mathrm{~h}$ and then stimulated with LPS for $1 \mathrm{~h}$. DCF-DA was added to the culture for 20 min during the final incubation, and intracellular ROS was subsequently measured using flow cytometric analysis of DCF fluorescence. (D) Cells transfected and incubated as in panel (C) were stimulated with LPS for $4 \mathrm{~h}$. Subsequently, total RNA was isolated and subjected to RT-PCR analysis. (E) Cells transfected and incubated as in panel (C) were stimulated with LPS for $12 \mathrm{~h}$, and the IL-6 released into the culture medium was subsequently measured. (F) Raw264.7 cells were transfected with control (siCont) or BLT2 (siBLT2) siRNAs. After 24 h, the cells were incubated in the presence or absence of LPS for $4 \mathrm{~h}$, and total RNA was then isolated and subjected RT-PCR analysis. All of the quantitative data are expressed as the mean \pm the SD, of three independent experiments. $* P<0.05, * * * P<0.005$ 
A

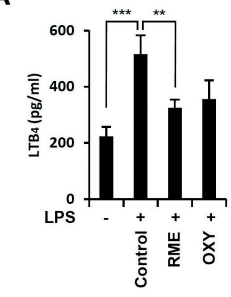

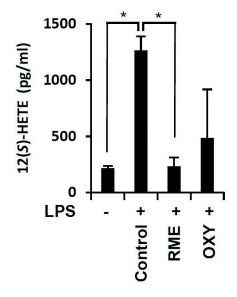

B

C

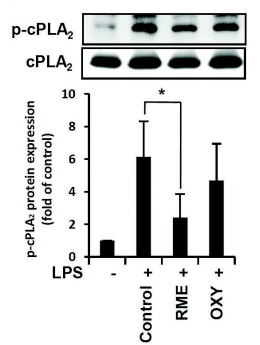

E

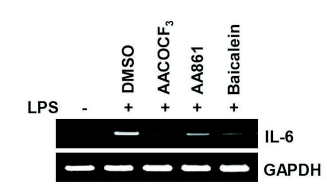

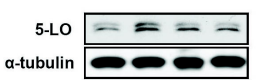

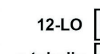

a-tubulin

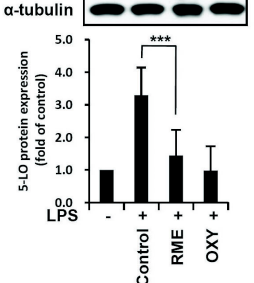

F

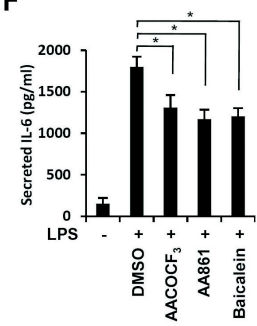

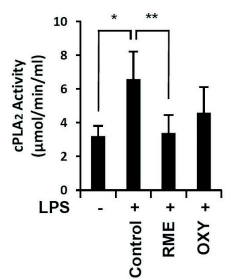

D

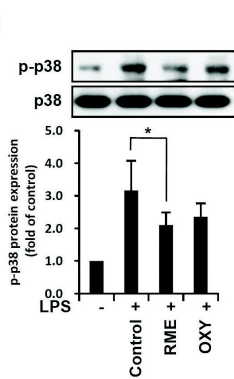

G

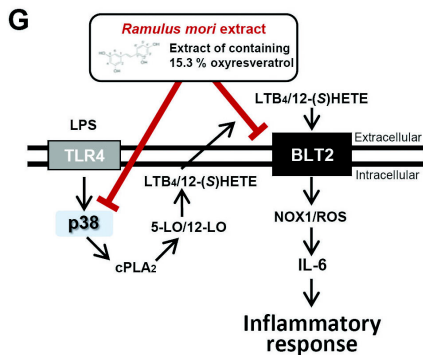

Fig. 4. RME inhibits LPS-induced BLT2 ligand production in Raw264.7 cells. (A) Raw264.7 cells were incubated with RME (47.5 $\mu \mathrm{g} / \mathrm{ml}$ ) or OXY $(7.3 \mu \mathrm{g} / \mathrm{ml})$ for $30 \mathrm{~min}$, and further incubated for $12 \mathrm{~h}$ in the presence or absence of LPS (100 ng/ml). The quantities of LTB 4 (left) and 12(S)-HETE (right) in the culture supernatants were measured by ELISA. (B) Raw264.7 cells were incubated for 30 min with RME or OXY, followed by $3 \mathrm{~h}$ incubation in the absence or presence of LPS. Next, the cells were assayed for cPLA ${ }_{2}$ activity by cPLA ${ }_{2}$ assay kit. (C) Raw264.7 cells were incubated for $30 \mathrm{~min}$ with RME or OXY, and then incubated for $3 \mathrm{~h}$ in the absence or presence of LPS. The cells were then evaluated for p-CPLA $, \mathrm{CPLA}_{2}, 5-\mathrm{LO}$ and $12-\mathrm{LO}$ protein levels by western blot assay (densitometry data also shown). (D) Cells were treated as detailed in $(\mathrm{C})$, and the cells were evaluated for p-p38 and p38 protein levels by Western blot (densitometry data also shown). (E) Raw264.7 cells were initially incubated for $30 \mathrm{~min}$ with AACOCF $3(20 \mu \mathrm{M})$, AA861 (10 $\mu \mathrm{M})$ or baicalein (20 $\mu \mathrm{M})$, followed by $4 \mathrm{~h}$ incubation in the presence or absence of LPS. Subsequently, total RNA was isolated and subjected to RT-PCR analysis. (F) Raw264.7 cells were initially incubated for $30 \mathrm{~min}$ with $\mathrm{AACOCF}_{3}$, AA861 or baicalein, and further incubated for $12 \mathrm{~h}$ in the presence or absence of LPS; the IL-6 released into the culture medium was then measured. $(G)$ The schematic representation of the mechanism of action by which RME exerts its inhibitory effects on LPS-induced inflammatory responses in Raw264.7 cells. All of the quantitative data are presented as the mean \pm the $\mathrm{SD}$, of three independent experiments. ${ }^{*} \mathrm{P}<0.05, * * \mathrm{P}<0.01, * * * \mathrm{P}<0.005$.

We observed that RME treatment markedly suppressed the p38 kinase activation (Fig. 4D). Under these experimental conditions, LPS-induced IL-6 expression was significantly decreased by 5 -LO (AA861), 12-LO (baicalein) and $\mathrm{CPLA}_{2}$ $\left(\mathrm{AACOCF}_{3}\right)$ inhibition in Raw264.7 cells (Fig. 4E and F). Together, these results suggest that RME suppresses the LPS-enhanced production of the BLT2 ligands $\mathrm{LTB}_{4}$ and $12(\mathrm{~S})-\mathrm{HETE}$, by attenuating the $\mathrm{p} 38-\mathrm{CPLA} 2-5-\mathrm{LO} / 12-\mathrm{LO}$ cascade.

In the present study, we demonstrated that RME inhibits LPS-induced IL-6 production. To analyze the detailed mode of action by which RME inhibits the LPS-induced IL-6 production, we evaluated the inhibitory effects of RME on each component (p38 kinase, cPLA $2, \mathrm{LTB}_{4}, 12(\mathrm{~S})-\mathrm{HETE}, \mathrm{BLT} 2$, and NOX1) in the
LPS signaling mediated IL-6 production. Our results conclusively identified the 'BLT2 ligand-BLT2'-linked axis as a target for the inhibitory action of RME. RME was shown to suppress the LPS-enhanced production of the BLT2 ligands, $\mathrm{LTB}_{4}$ and 12(S)-HETE, by attenuating the p38-CPLA $2-5-\mathrm{LO} / 12-\mathrm{LO}$ cascade (Figs. 4A-D). Specifically, we speculate that RME targets the p38 kinase (Fig. 4D), thus attenuating the subsequent activation of downstream components ( $\mathrm{CPLA}_{2}, 5-\mathrm{LO}$, and 12-LO) (Figs. $4 \mathrm{~B}$ and C). In fact, previous studies have shown that that LPS-induced IL-6 production in macrophages is regulated through p38 kinase and p38 kinase-mediated cPLA ${ }_{2}$ activation, thus pointing to p38 kinase as a principal contributor to the LPS-induced production of IL-6 in macrophages $(32,33)$. 
Additionally, we also observed that RME suppressed the BLT2 expression levels (both the mRNA and protein levels) (Figs. 2C and D). Thus, altogether, RME appears to suppress the production of BLT2 ligands as well as the BLT2 expression.

LPS-induced IL-6 production has been suggested to play a role in the development of endotoxic or septic lung inflammation. Indeed, the production of IL- 6 was shown to be a hallmark of sepsis, with high levels of this cytokine in affected individuals being associated with mortality $(34,35)$. Considering that BLT2 is a crucial mediator of LPS signaling-induced IL-6 production in macrophages (as summarized in Fig. 4G), our findings may point to the potential development of RME-based herbal medications being therapeutic for patients with endotoxic or septic shock. Additionally, BLT2 has been implicated in other inflammatory pathogenic conditions as well, including asthmatic airway inflammation and cancer, thus further expanding the potential clinical use of this herbal medicine as being applicable to various BLT2-associated inflammatory diseases.

In summary, our results demonstrated that RME suppresses the IL-6 production in LPS-activated Raw264.7 cells, and that the 'BLT2 ligand-BLT2'-linked autocrine inflammatory axis is one of the targets of the anti-inflammatory activity of RME. The elucidation of this mechanism provides significant insight into the anti-inflammatory activity of $R$. mori.

\section{MATERIALS AND METHODS}

Detailed information is described in online Supplementary Data.

\section{ACKNOWLEDGEMENTS}

This work was supported by the High Value-added Food Technology Development Program (313028-3) of the Ministry of Agriculture, Food and Rural Affairs, Republic of Korea, and the BK21 Plus Program (College of Life Sciences and Biotechnology, Korea University). This work was also supported by a Bio \& Medical Technology Development Program grant (2012M3A9C5048709, 2012M3A9C1053532) and a Mid-career Researcher Program grant (2015R1A2A1A15053311) through the National Research Foundation (NRF) funded by the Ministry of Science, Information and Communication Technologies (ICT) and Future Planning, Republic of Korea and the Basic Science Research Program (2015R1D1A1A01057757) through the NRF funded by the Ministry of Education.

\section{REFERENCES}

1. Nomura T (2001) [Chemistry and biosynthesis of prenylflavonoids]. Yakugaku Zasshi 121, 535-556

2. Zhou J, Li SX, Wang W et al (2013) Variations in the levels of mulberroside $A$, oxyresveratrol, and resveratrol in mulberries in different seasons and during growth. Scientific World Journal 2013, 380692
3. Lee HS, Kim DH, Hong JE, Lee JY and Kim EJ (2015) Oxyresveratrol suppresses lipopolysaccharide-induced inflammatory responses in murine macrophages. Hum Exp Toxicol 34, 808-818

4. Lorenz P, Roychowdhury S, Engelmann M, Wolf G and Horn TF (2003) Oxyresveratrol and resveratrol are potent antioxidants and free radical scavengers: effect on nitrosative and oxidative stress derived from microglial cells. Nitric Oxide 9, 64-76

5. Hwang D, Jo SP, Lee J, Kim JK, Kim KH and Lim YH (2015) Antihyperlipidaemic effects of oxyresveratrol-containing Ramulus mori ethanol extract in rats fed a high-cholesterol diet. Journal of Functional Foods 19, 353-362

6. Eo HJ, Park JH, Park GH et al (2014) Anti-inflammatory and anti-cancer activity of mulberry (Morus alba L.) root bark. BMC Complement Altern Med 14, 200

7. Wang $\mathrm{W}, \mathrm{Zu} \mathrm{Y}, \mathrm{Fu} \mathrm{Y}$ and Efferth $\mathrm{T}$ (2012) In vitro antioxidant and antimicrobial activity of extracts from Morus alba L. leaves, stems and fruits. Am J Chin Med 40, 349-356

8. Lim HJ, Jin HG, Woo ER, Lee SK and Kim HP (2013) The root barks of Morus alba and the flavonoid constituents inhibit airway inflammation. J Ethnopharmacol 149, 169175

9. Zhang Z, Jin J and Shi L (2008) Protective function of cis-mulberroside $\mathrm{A}$ and oxyresveratrol from Ramulus mori against ethanol-induced hepatic damage. Environ Toxicol Pharmacol 26, 325-330

10. Zhang $Z$ and Shi L (2010) Anti-inflammatory and analgesic properties of cis-mulberroside A from Ramulus mori. Fitoterapia 81, 214-218

11. $\mathrm{Xu} \mathrm{L}$, Yang $\mathrm{F}$, Wang J, Huang $\mathrm{H}$ and Huang $\mathrm{Y}$ (2015) Anti-diabetic effect mediated by Ramulus mori polysaccharides. Carbohydr Polym 117, 63-69

12. Yokomizo $T$, Kato $K$, Hagiya $H$, Izumi $T$ and Shimizu $T$ (2001) Hydroxyeicosanoids bind to and activate the low affinity leukotriene B4 receptor, BLT2. J Biol Chem 276, 12454-12459

13. Cho KJ, Seo JM, Lee MG and Kim JH (2010) BLT2 Is upregulated in allergen-stimulated mast cells and mediates the synthesis of Th2 cytokines. J Immunol 185, 6329-6337

14. Cho KJ, Seo JM, Shin Y et al (2010) Blockade of airway inflammation and hyperresponsiveness by inhibition of BLT2, a low-affinity leukotriene B4 receptor. Am J Respir Cell Mol Biol 42, 294-303

15. Mathis SP, Jala VR, Lee DM and Haribabu B (2010) Nonredundant roles for leukotriene B4 receptors BLT1 and BLT2 in inflammatory arthritis. J Immunol 185, 3049-3056

16. Park GS and Kim JH (2015) LPS Up-Regulates ICAM-1 Expression in Breast Cancer Cells by Stimulating a MyD88BLT2-ERK-Linked Cascade, Which Promotes Adhesion to Monocytes. Mol Cells 38, 821-828

17. Park GS and Kim JH (2015) Myeloid differentiation primary response gene 88-leukotriene B4 receptor 2 cascade mediates lipopolysaccharide-potentiated invasiveness of breast cancer cells. Oncotarget 6, 5749-5759

18. Park J, Park SY and Kim JH (2016) Leukotriene B4 receptor-2 contributes to chemoresistance of SK-OV-3 ovarian cancer cells through activation of signal transducer 
and activator of transcription-3-linked cascade. Biochim Biophys Acta 1863, 236-243

19. Singh VP, Patil CS, Jain NK, Singh A and Kulkarni SK (2003) Effect of nimesulide on acetic acid- and leukotriene-induced inflammatory bowel disease in rats. Prostaglandins Other Lipid Mediat 71, 163-175

20. Kim GY, Lee JW, Ryu HC, Wei JD, Seong CM and Kim JH (2010) Proinflammatory cytokine IL-1beta stimulates IL-8 synthesis in mast cells via a leukotriene B4 receptor 2-linked pathway, contributing to angiogenesis. J Immunol $184,3946-3954$

21. Lee AJ, Cho KJ and Kim JH (2015) MyD88-BLT2-dependent cascade contributes to LPS-induced interleukin-6 production in mouse macrophage. Exp Mol Med 47, e156

22. Gabay C (2006) Interleukin-6 and chronic inflammation. Arthritis Res Ther 8 Suppl 2, S3

23. Kwon DJ, Bae YS, Ju SM, Youn GS, Choi SY and Park J (2014) Salicortin suppresses lipopolysaccharide-stimulated inflammatory responses via blockade of NF-kappaB and JNK activation in RAW 264.7 macrophages. BMB Rep 47, 318-323

24. Hoyer FF, Albrecht L, Nickenig G and Muller C (2012) Selective inhibition of leukotriene receptor BLT-2 reduces vascular oxidative stress and improves endothelial function in ApoE-/- mice. Mol Cell Biochem 359, 25-31

25. Maitra U, Singh N, Gan L, Ringwood L and Li L (2009) IRAK-1 contributes to lipopolysaccharide-induced reactive oxygen species generation in macrophages by inducing NOX-1 transcription and Rac1 activation and suppressing the expression of antioxidative enzymes. J Biol Chem 284, 35403-35411

26. Kim JS, Yeo S, Shin DG et al (2010) Glycogen synthase kinase 3beta and beta-catenin pathway is involved in toll-like receptor 4-mediated NADPH oxidase 1 expres- sion in macrophages. FEBS J 277, 2830-2837

27. Kim EY, Seo JM, Kim C, Lee JE, Lee KM and Kim JH (2010) BLT2 promotes the invasion and metastasis of aggressive bladder cancer cells through a reactive oxygen species-linked pathway. Free Radic Biol Med 49, 10721081

28. Powell WS, Gravel S, Khanapure SP and Rokach J (1999) Biological inactivation of 5-oxo-6,8,11,14-eicosatetraenoic acid by human platelets. Blood 93, 1086-1096

29. Funk CD (2001) Prostaglandins and leukotrienes: Advances in eicosanoid biology. Science 294, 1871-1875

30. Leslie CC (1997) Properties and regulation of cytosolic phospholipase A2. J Biol Chem 272, 16709-16712

31. Qi HY and Shelhamer JH (2005) Toll-like receptor 4 signaling regulates cytosolic phospholipase A2 activation and lipid generation in lipopolysaccharide-stimulated macrophages. J Biol Chem 280, 38969-38975

32. Uozumi N, Kita Y and Shimizu T (2008) Modulation of lipid and protein mediators of inflammation by cytosolic phospholipase A2alpha during experimental sepsis. J Immunol 181, 3558-3566

33. Wang X, Xue H, Xu Q et al (2008) p38 kinase/cytosolic phospholipase A2/cyclooxygenase-2 pathway: a new signaling cascade for lipopolysaccharide-induced interleukin-1beta and interleukin-6 release in differentiated U937 cells. Prostaglandins Other Lipid Mediat 86, 61-67

34. Rau S, Kohn B, Richter C et al (2007) Plasma interleukin-6 response is predictive for severity and mortality in canine systemic inflammatory response syndrome and sepsis. Vet Clin Pathol 36, 253-260

35. Song R, Kim J, Yu D, Park C and Park J (2012) Kinetics of IL-6 and TNF-alpha changes in a canine model of sepsis induced by endotoxin. Vet Immunol Immunopathol 146, 143-149 